\title{
Well connection optimization in integrated subsurface and surface facilities: an industrial case study
}

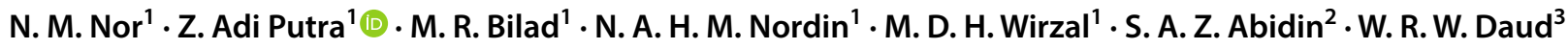

Received: 24 January 2019 / Accepted: 6 May 2019 / Published online: 14 May 2019

(c) The Author(s) 2019

\begin{abstract}
Optimizations of oil productions in current practices have been done separately in both subsurface and surface facilities. This approach might not lead to an optimum state when the whole system is interconnected. In this work, a superstructure model of an existing integrated subsurface and surface facilities was developed and evaluated to maximize the oil productions. In subsurface facilities, well performance data were taken from PROSPER well simulations. Operating variables such as downstream pressures of choke valves and gas lift flowrates, which influence the flow of oil, gas, and water from reservoirs, were considered. Based on these variables, performance correlations of the wells were then developed. On the evaluated surface facility, three pressure levels of vessels on the platform (high-, medium-, and low-pressure vessels) and their current designs were also considered. The optimization result shows that to reach optimum oil production, one well should be connected to the high-pressure vessel, four wells to the medium-pressure vessel, and the rest goes to the low-pressure vessel. Several choke valves and gas lift flowrates also need to be adjusted accordingly.
\end{abstract}

Keywords Surface and subsurface optimization · Oil production · Choke valves · Gas lifts · Wellhead connections

\section{Introduction}

Nowadays, oil and gas are still the main energy resources and they will continue to be one of the major resources in the near future. A common oil and gas production system consists of (1) one or more reservoirs, (2) a production gathering system which consists of wells, flow lines, and pipelines, (3) platform processing, as well as (4) export facilities (Abidin and Hussein 2014). The first two are commonly referred to as subsurface facility, while the latter two are surface facilities. Figure 1a, b shows a typical oil and gas production system and a Christmas tree that connects a well to the surface facility, respectively.

Optimizing production of oil is very challenging as it involves various types of processes and at the same time,

Z. Adi Putra

zulfan.adiputra@utp.edu.my

1 Chemical Engineering Department, Universiti Teknologi PETRONAS, 32610 Seri Iskandar, Perak, Malaysia

2 PETRONAS Group Technical Solutions Process Simulation and Optimization, 500050 Kuala Lumpur, Malaysia

3 Chemical and Process Engineering Department, Universiti Kebangsaan Malaysia, 43600 Bangi, Selangor, Malaysia its inherent dynamic situations (Shah and Mishra 2013; Izadmehr et al. 2018). According to Rahmawati et al. (2012), integrated modeling and optimization is an important approach in the petroleum industry, especially for field development and continuous asset-management evaluations. Optimization in oil and gas industries can be composed into three main topics: drilling and well completion, reservoir management and production optimization, as well as operations and maintenance (Sarra et al. 2015).

Conventional modeling approach consists of applications of many stand-alone models correlating various fundamentals of a petroleum asset in a silo-model approach. This includes reservoir models, well models, surface process models, export and sales models, and economic models. Optimizing these models separately can only accomplish production increment of up to one percent (Abidin and Hussein 2014). On the other hand, integrated modeling of subsurface and surface facilities potentially offers large untapped benefits. However, integration of those silo-models to optimize the overall system performance faces many technological challenges (Rahmawati 2012).

Researchers have conducted various integrated modeling approaches for oil productions. Bailey and Couet (2005) and Litvak et al. (2011) studied complex petroleum field projects 


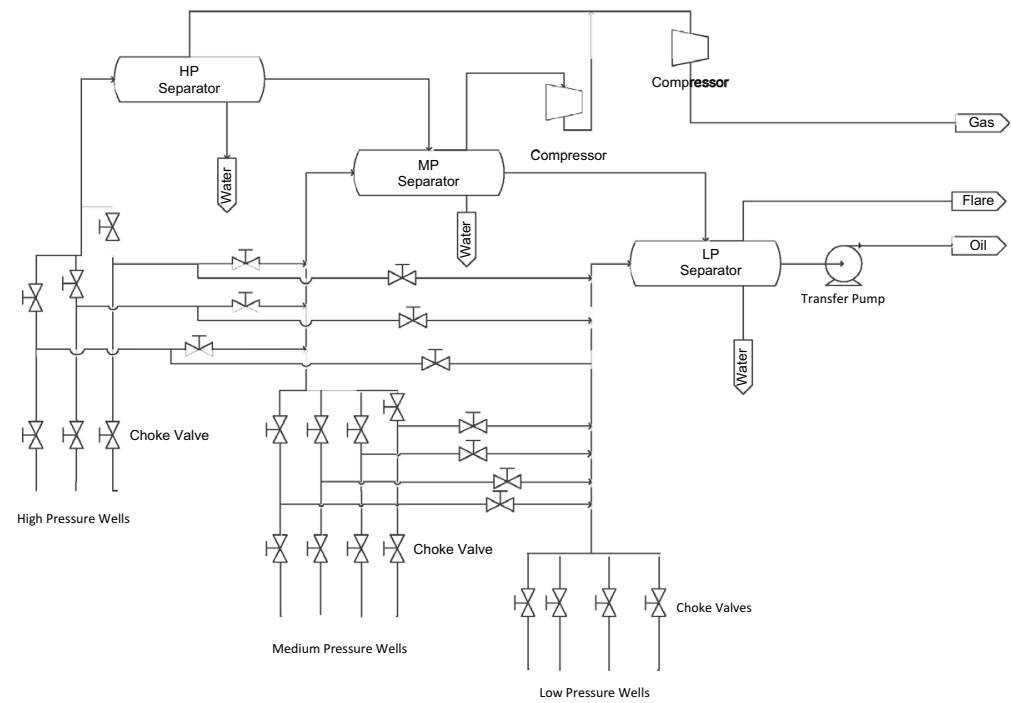

(a)

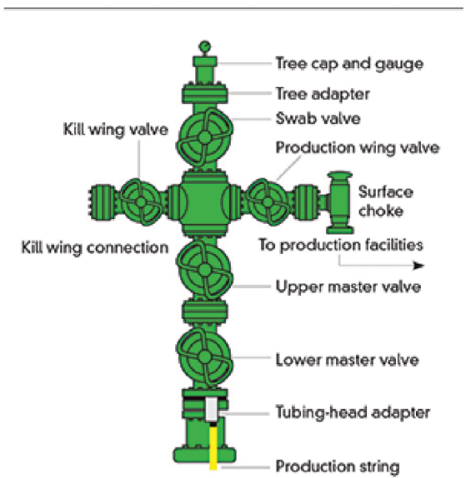

(b)

Fig. 1 a Typical subsurface and surface connections and $\mathbf{b}$ typical surface choke valve in a Christmas tree (Izadmehr et al. 2018)

by simplifying the surface process facility prior to applying uncertainty analysis. Sarra et al. (2015) combined surface production, subsurface models, and process models in a single management tool called rabbit ${ }^{\mathrm{TM}}$, Risked Algorithms for Biogenetical Balance Integration. The combined model considers the transient behaviors in the pipeline. Meanwhile, Queipo et al. (2003) modeled cumulative oil production where they considered tubing, choke, and pipeline diameter, as well as oil flowrate as the variables. Then they solved it using a nonlinear programming technique such as sequential quadratic programming (SQP). Rahmawati et al. (2012) integrated SENSOR (reservoir modeling), PROSPER (well modeling), and a simple surface facility model using HYSYS in a platform called Pipe-It ${ }^{\circledR}$. Escalona et al. (2014) developed an integrated subsurface-surface model to support future exploitation plan by considering a constant oil production and an aggressive infill drilling. These works mainly focused in optimizing the subsurface facilities without considering the operating conditions of surface facilities such as pressures of the separation vessels. Zainal et al. (2018) combined an in-house process simulation software called iCON with PROSPER well models to optimize the operating conditions (e.g., pressures) of surface facilities to maximize liquid productions. Nonetheless, these previous works considered only fixed connections between the wells and the separation vessels on the surface facilities. However, there are various connections between the wells and the separation vessels in the real-surface facilities. Hence, optimizing this connection remains a trial and error practice.

In this work, pipe connections of several existing wells and a surface facility are optimized to increase oil productions. Regression models of wells performances are first developed based on the data provided by the PROSPER models of the wells. These models are then mapped against the existing pressures of the vessels on the surface facility. This mapping forms a superstructure consisting of all possible connections between the wells and the vessels. Optimization problem is then formulated to maximize the oil production with existing equipment designs (e.g., lines, control valves, and pumps) as the constraints. Several actual oil and gas wells were used as the case study. Due to confidentiality reason, the names of the wells and their data are modified.

\section{Methodology}

The methodology of this project is illustrated in Fig. 2. Firstly, the performances of the wells need to be understood before creating a superstructure that connect the wells $(i)$ and the vessels $(j)$ on the surface facility. For this purpose, well performance data from PROSPER simulations and equipment datasheets were collected and analyzed. The analysis resulted in performance correlations of each well that links the oil rate productions as a function of downstream pressures of choke valves or openings of choke valves $\left(P_{i}\right)$ and gas lifts $\left(G_{i}\right)$. Choke valve is used to adjust the fluid flowrate coming from the well. Gas lift is the amount of gas injected into the reservoir to reduce oil viscosities, which then reduces the oils' flow resistance and thus increases the oil production rates.

Secondly, oil production rates $\left(F_{j}\right)$ of each well were evaluated using an in-house process simulation software (iCON) for various pressure vessels $\left(F_{i, j}\right)$. The oil flowrates from each vessel $\left(F_{j}\right)$ were subjected to the existing designs of the pipes, 


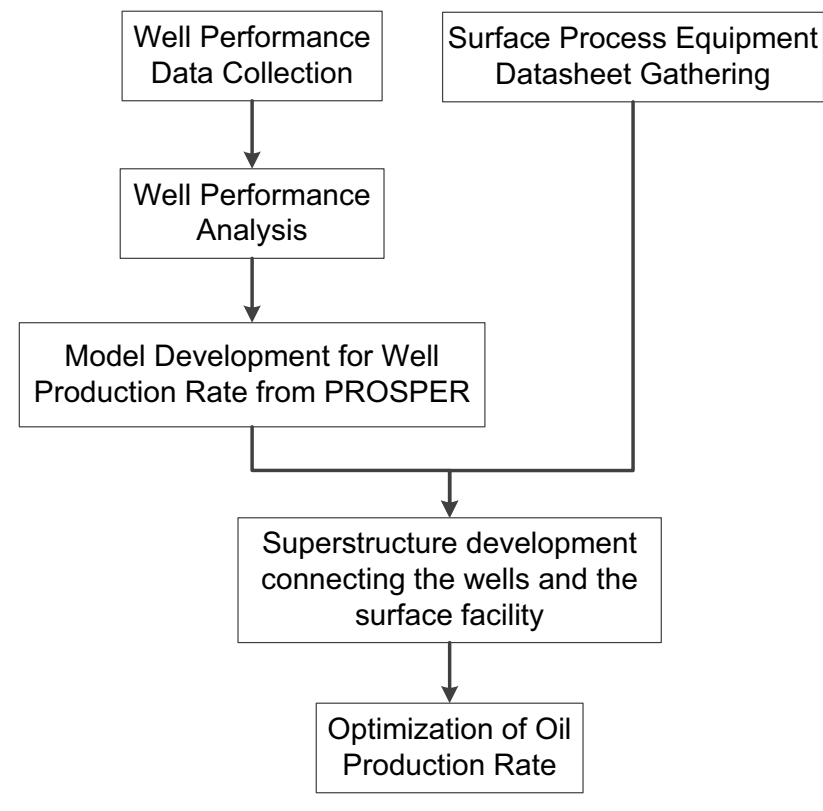

Fig. 2 Methodology

control valves, and transfer pumps $\left(F_{j, \max }\right)$. Finally, a superstructure was developed. The superstructure consisted of all possible connections mapping the wells' performances to the available pressures of the vessels in the surface facility. This mapping was then formulated as an optimization problem with the objective to maximize the oil production. The optimization was done by utilizing only the existing facilities without requiring additional capital investment (CAPEX). Hence, existing equipment designs of the facility, such as pipeline sizes, control valves, and transfer pumps, were used as the optimization constraints.

In this work, the performances of four wells are affected solely by the downstream pressures of choke valves pressures. In this regard, linear regressions to calculate the oil, water, and gas with various downstream pressures of the choke valves are formulated. On the other hand, five other wells are dependent on both downstream pressures of choke valves pressures and gas lifts. In this case, some nonlinearities are included in the regressions, such as the multiplication of choke valves' downstream pressures and gas lifts, and the quadratic forms of these variables. The relationship between the downstream pressures of the choke valves and the pressure of the vessels in the surface facility for each well is also evaluated. If both pressures are too low, the oil can then evaporate in the vessel, which then reduces the oil production. Hence, there is a minimum pressure allowed in the process vessels.

The objective of the optimization is to maximize the total oil production from the vessels on the surface facility $\left(F_{j}\right)$ as follows: $\max \sum_{j \in J}^{m} F_{j}$

Subject to:

$$
\begin{aligned}
& 50 \leq P_{i} \leq 500 \quad \forall i \in I \\
& 0.2 \leq G_{i} \leq 2 \quad \forall i \in I \\
& 0 \leq F_{j} \leq F_{j, \max } \quad \forall j \in J \\
& F_{i, j}=f\left(P_{i}, G_{i}\right) \quad \forall i \in I \\
& F_{j}=\sum_{i \in I}^{n} F_{i, j} \quad \forall j \in J
\end{aligned}
$$

Following practical constrains are considered during the optimization processes. The performances of four wells are affected solely by the downstream pressures of choke valves. Hence, linear regressions were formulated to calculate the oil, water, and gas with various downstream pressures of the choke valves. On the other hand, five other wells are dependent on both downstream pressures of choke valves and gas lifts. Therefore, some nonlinearities were included in the regressions, such as the multiplication of choke valves' downstream pressures and gas lifts and the quadratic forms of these variables. The relationship between the downstream pressures of the choke valves and the pressure of the vessels in the surface facility for each well was also evaluated and considered. For instance, if both pressures were too low, the oil may evaporate in the vessel, which then reduced the oil production. Hence, there was a minimum pressure value as a constrain allowed in each process vessels.

\section{Results and discussions}

\section{Well performances}

Figure 3 a shows the relations between oil production rate as a function of the pressure of choke valves for Wells A, D, $\mathrm{H}$, and I. It can be observed that the oil production increases as the downstream pressure of the choke valve decreases. This is due to the constant pressure of the reservoir. As the downstream pressure of the choke valve decreases, a higher pressure gradient or pressure drop is created, and hence, a higher flowrate through the well. This finding is in line with other reports (Van Der and Muntinga 1999) and can be justified since the pressure drop is normally used to regulate the production rate. 


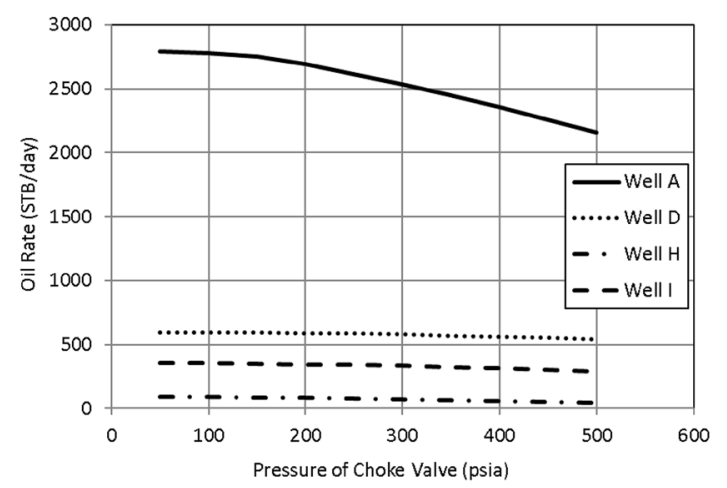

(a)

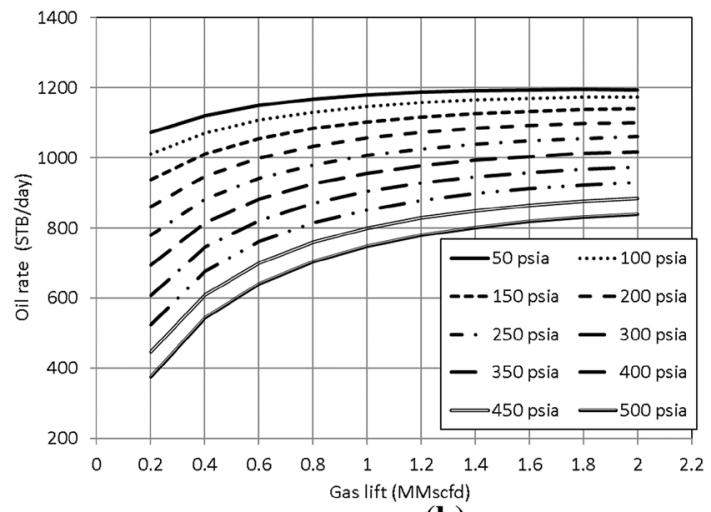

(b)

Fig. 3 Performances of wells as a function of $\mathbf{a}$ downstream pressures of choke valves and $\mathbf{b}$ downstream pressure of choke valves and gas lifts

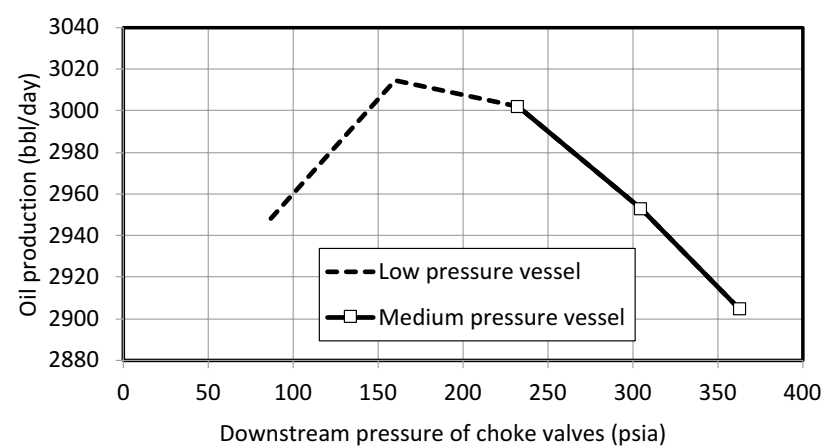

Fig. 4 Variation of oil productions from Well A considering both choke valves and pressures of vessels on the platform

The performances of the other wells ( B, E, F, and K) are affected by both downstream pressures of choke valves and gas lifts (Fig. 3b). The effect of downstream pressure of choke valves is like the one on Wells A, D, H, and I. In addition, Fig. $3 \mathrm{~b}$ also shows that increasing the amount of gas lift of up to about 1.4 MMscfd increases the oil production. Beyond 1.4 MMscfd, there is no effect of gas lift on the oil production. The other three wells also show the same steady oil production up to gas lift of about 1.4 MMscfd.

The correlation between the oil production of a well and the gas lift flowrates is important in optimizing oil rate productions, as suggested by Shedid and Yakoot (2016). The result shows the advantage of increasing gas lifts on well's productivity. It is also showing the phenomena of "over injection," where little or no benefit is obtained by increasing the gas lifts. However, in extreme cases where there is a high water cut, increasing gas lift may lead to a reduction in well productivities. Under such situations, water having the lower viscosity flows much faster than the oil. Hence, the oil production is reduced. Based on the performance data in Fig. 3, polynomial regression models of the wells were formulated. For the wells that use both choke valves and gas lifts, the interaction between the two variables was considered in the model. This makes the models to become nonlinear. From data in Fig. 3, maximum oil production can be obtained at the lowest pressure of the choke valves.

Figure 4 shows oil productions from Well A when the above regression models were used and simulated to see the effect of pressure levels in the surface facility. The process simulation models demonstrate that the reduction of the choke valve pressures is limited by the pressure on the surface vessel. Below a certain pressure, the oil evaporates and hence the oil production reduces. This practical insight is lacking from previous works that focused more on the subsurface facilities while over-simplifying the operating conditions of the surface facility. This phenomenon is well known for the engineers working on surface facilities, but not known to those in the subsurface facilities.

\section{Superstructure optimization}

As shown in Fig. 1, there are three separator vessels on the surface facility. Each of them operates at different pressures, namely high (290 psia), medium (145 psia), and low (72.5 psia). To establish the superstructure connections between the wells and the vessels, both the performance curves of the wells and the insights from the variations of pressures of the vessels were used. Based on this information, the wells with the stable flowrate variations, and insensitive to the choke valve pressures (about $<10 \%$ ), could be connected to the higher-pressure vessels. On the other hand, the unstable wells (characterized by very steep declines of oil flowrate $>10 \%$ deviation) should be sent to the lower-pressure vessels to maximize their production. Based on those criteria, the mappings of the wells and the separator vessels were established and shown in Table 1. The developed superstructure based on Table 1 is shown in Fig. 5a.

After establishing the superstructure, we solved the optimization problem shown in Eqs. (1)-(6). The optimization 
Table 1 Establishing the well connections to the pressure vessels based on their pressures

\begin{tabular}{llll}
\hline Well & \multicolumn{2}{l}{ Separator pressure selection } \\
\cline { 2 - 4 } & Low pressure & $\begin{array}{l}\text { Medium pres- } \\
\text { sure }\end{array}$ & High pressure \\
\hline Well A & Yes & Yes & \\
Well B & Yes & Yes & Yes \\
Well D & Yes & Yes & \\
Well E & Yes & Yes & \\
Well $\mathrm{F}$ & Yes & & Yes \\
Well H & Yes & Yes & \\
Well I & Yes & Yes & \\
Well K & Yes & Yes & \\
\hline
\end{tabular}

problem was formulated and solved in general algebraic modeling systems (GAMS). Tables 2 and 3 show the oil production rates from each vessel at optimum choke valve pressures and the gas lifts, respectively. The optimum configuration is shown in Fig. 5b. The optimization model has successfully identified the optimum downstream choke valves pressures and the gas lifts flowrates of to maximize the oil production rate. It can be seen that under the optimum connection and conditions, Well A, for example, should be connected to the medium-pressure vessel. This result is in agreement with the insight found in Fig. 4, where it can be seen that if the choke pressure of Well A is reduced further, the oil production decreases. Table 3 also shows that the choke valve pressure of Well A is lower than the maximum pressure found in Fig. 4. This is because of the existing design constraints (e.g., pipe size, control valves, and pumps) of the MP vessel that limit this choke valve pressure to be reduced further.

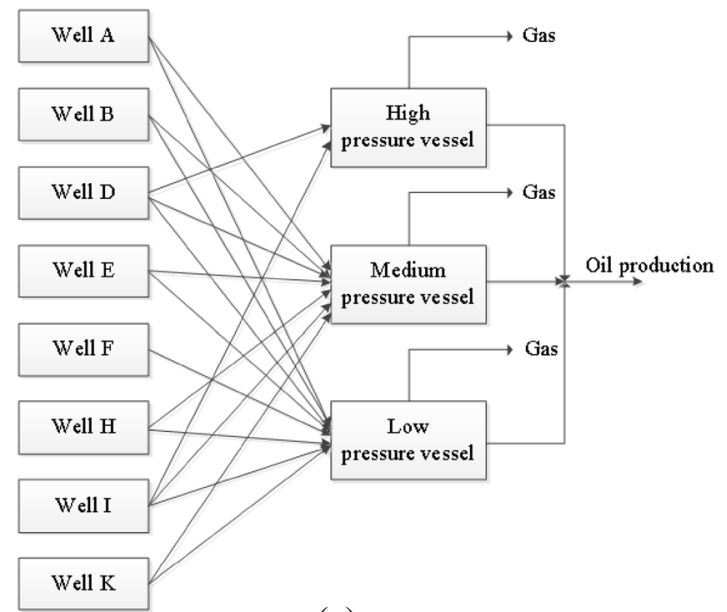

(a)
Table 2 Oil flowrate produced from each separation vessel

\begin{tabular}{lcc}
\hline Type of separator & Capacity $\left(\mathrm{m}^{3} / \mathrm{h}\right)$ & $\begin{array}{l}\text { Optimum } \\
\text { capacity } \\
\left(\mathrm{m}^{3} / \mathrm{h}\right)\end{array}$ \\
\hline High-pressure vessel & 3.600 & 3.600 \\
Medium-pressure vessel & 22.400 & 22.400 \\
Low-pressure vessel & 22.400 & 22.400 \\
\hline
\end{tabular}

Table 3 Optimum process variables to maximize the oil production

\begin{tabular}{lll}
\hline Type of well & Optimum pressure (psig) & $\begin{array}{l}\text { Optimum gas } \\
\text { lift (MMscfd) }\end{array}$ \\
\hline Well A & 255 & - \\
Well B & 493 & 0.200 \\
Well D & 480 & - \\
Well E & 481 & 0.201 \\
Well F & 500 & 0.201 \\
Well H & 500 & - \\
Well I & 500 & - \\
Well K & 395 & 0.201 \\
\hline
\end{tabular}

\section{Conclusion}

In this paper, an integrated optimization of subsurface and surface facility has been conducted. Well performance data were taken from PROSPER simulation models, which then became the basis for development of performance regression models. These models were then used further to evaluate the oil production when these wells were connected to existing pressure vessels on the surface facility. The results of this initial assessment were used as the basis for developing a

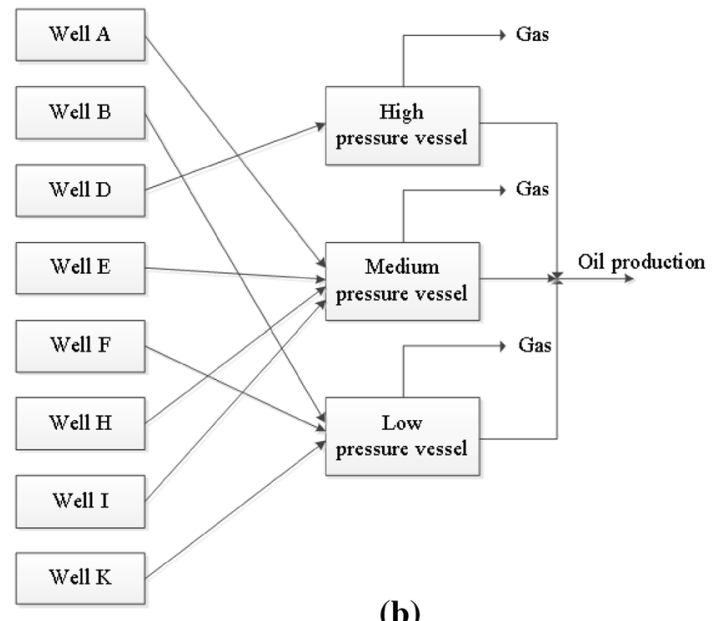

(b)

Fig. 5 Connections from wells to the pressure vessels, a the developed superstructure of the wells and pressure vessels, and $\mathbf{b}$ the optimum connection 
superstructure connecting the subsurface and surface facility. The optimization problem based on this superstructure was then solved, yielding an optimum connection between the subsurface (the wells) and the surface (pressure vessels) facilities. The results of the optimum connections have been evaluated and are in agreement with the practical insights where the reduction in the downstream pressures of choke valves is limited to a certain value, and also to the respective design constraints. This optimum connection will serve as a good starting point for future optimization involving more rigorous process simulation.

Open Access This article is distributed under the terms of the Creative Commons Attribution 4.0 International License (http://creativecommons.org/licenses/by/4.0/), which permits unrestricted use, distribution, and reproduction in any medium, provided you give appropriate credit to the original author(s) and the source, provide a link to the Creative Commons license, and indicate if changes were made.

\section{References}

Abidin SA, Hussein N (2014) Integrated optimisation for surface and sub surface towards maximising production. In: Offshore technology conference

Bailey WJ, Couet B (2005) Field optimization tool for maximizing asset value. SPE Reserv Eval Eng 8:7-21. https://doi. org/10.2118/87026-PA

Escalona J, Figueroa A, Lopez L (2014) Fully compositional integrated subsurface-surface modeling: Carito production unit case. Society of Petroleum Engineers, Kuala Lumpur

Izadmehr M, Daryasafar A, Bakhshi P et al (2018) Determining influence of different factors on production optimization by developing production scenarios. J Pet Explor Prod Technol 8:505-520. https ://doi.org/10.1007/s13202-017-0351-1

Litvak ML, Onwunalu JE, Baxter J (2011) Field development optimization with subsurface uncertainties. Society of Petroleum Engineers, Kuala Lumpur

Queipo NV, Zerpa LE, Goicochea JV et al (2003) A model for the integrated optimization of oil production systems. Eng Comput 19:130-141. https://doi.org/10.1007/s00366-003-0255-1

Rahmawati SD (2012) Integrated field modeling and optimization. Norges teknisk-naturvitenskapelige universitet, Fakultet for informasjonsteknologi, matematikk og elektroteknikk, Institutt for teknisk kybernetikk

Rahmawati SD, Whitson CH, Foss B, Kuntadi A (2012) Integrated field operation and optimization. J Pet Sci Eng 81:161-170. https://doi. org/10.1016/j.petrol.2011.12.027

Sarra A, Ritondale S, Viadana G et al (2015) Integrated optimization of surface assets with fluid-dynamic check workflow: a real case application. In: Offshore mediterranean conference

Shah N, Mishra P (2013) Oil production optimization: a mathematical model. J Pet Explor Prod Technol 3:37-42. https://doi. org/10.1007/s13202-012-0040-z

Shedid SA, Yakoot MS (2016) Simulation study of technical and feasible gas lift performance. Int J Pet Sci Eng 10:21-44

Van Der ZM, Muntinga JH (1999) The effects of production rate and choke size on emulsion stability. Lima, Peru

Zainal SA, Daud WR, Putra ZA, Nor N (2018) Integrated constraints optimization for surface and sub-surface towards CAPEX free maximizing production. IOP Conf Ser Mater Sci Eng 458:012045. https://doi.org/10.1088/1757-899X/458/1/012045

Publisher's Note Springer Nature remains neutral with regard to jurisdictional claims in published maps and institutional affiliations. 